\title{
Emodin exerts antitumor effects in ovarian cancer cell lines by preventing the development of cancer stem cells via epithelial mesenchymal transition
}

\author{
HEMING LONG, HONGMEI CHEN, JUN YAN and HAIYAN CHENG \\ Department of Internal Medicine - Oncology, First Affiliated Hospital of Gannan Medical University, \\ Ganzhou, Jiangxi 341000, P.R. China
}

Received March 24, 2021; Accepted September 17, 2021

DOI: 10.3892/ol.2022.13215

\begin{abstract}
Ovarian cancer has the worst prognosis among all types of gynecological malignancies and patients are often diagnosed at an advanced stage with distant metastasis. In the present study, it was found that emodin, a small molecular chemical drug derived from natural plants, has antitumor effects on ovarian cancer cells. Emodin induced cytotoxicity and inhibited proliferation in the ovarian cancer cell lines, SK-OV-3, A2780 and PA-1. In addition, emodin inhibited the migration and invasion abilities of the ovarian cancer cells by inhibiting epithelial-mesenchymal transition (EMT), which was evidenced by the downregulation of $\mathrm{N}$-cadherin and vimentin, and the upregulation of E-cadherin protein expression levels. When a subcutaneous xenograft SK-OV-3 tumor mouse model was used, emodin notably reduced the tumor growth rate and inhibited tumor cell proliferation. Furthermore, mechanical analysis revealed that emodin markedly inhibited EMT and reduced the stemness of tumor cells, which was evidenced by the decrease in the protein expression of CD133 and Oct4. Pulmonary metastasis of the ovarian cancer cells was significantly suppressed in the tumor mouse model by the administration of emodin. In addition, flow cytometry analysis indicated that emodin significantly reduced the proportion of ovarian cancer stem-like cells in metastatic lung tissues. In conclusion, emodin, a potent inhibitor of EMT, could serve as a potential candidate for ovarian cancer therapy.
\end{abstract}

Correspondence to: Professor Haiyan Cheng, Department of Internal Medicine - Oncology, First Affiliated Hospital of Gannan Medical University, 23 Qingnian Road, Ganzhou, Jiangxi 341000, P.R. China

E-mail: chhaiyan123@sina.com

Key words: emodin, metastasis, epithelial-mesenchymal transition, ovarian cancer, cancer stem cells

\section{Introduction}

Ovarian cancer is a malignant carcinoma that is usually diagnosed at a late stage, by which time metastasis can be observed at distant sites, including the lungs, liver and lymph $(1,2)$. Ovarian cancer usually develops from the ovarian surface epithelium or from serous intra-epithelial carcinoma (3). With the advancement of medical technology, debulking surgery and cis-platinum-based chemotherapy are the major therapeutics for ovarian cancer. However, the outcome of debulking surgery is dependent on surgical skill and the genetic features of the tumor $(4,5)$. Debulking surgery combined with cis-platinum based chemotherapy can lead to remission in patients with ovarian cancer; however, the majority of patients suffer from cancer resistance, metastasis and relapse (6,7). Following relapse, the outcome of chemotherapy declines significantly, with rapid disease progression and drug resistance. Furthermore, genetic alterations in cancer cells, which are widely investigated for drug resistance and cancer metastasis, result in drug inactivation, enhanced DNA repair mechanisms and changes in the intracellular pathways (8). However, cancer metastasis and relapse remain important obstacles for ovarian cancer therapy, and novel and efficient therapeutics for ovarian cancer are urgently required.

Epithelial-mesenchymal transition (EMT) plays key roles in cancer proliferation and distant metastasis $(9,10)$. Various signaling pathways have been associated with extracellular cues, such as TGF- $\beta$ and play important roles in reprogramming gene expression during EMT $(11,12)$. Furthermore, cancer stem cells (CSCs), which are characterized by the markers, CD133 (also known as prominin-1) and octamer binding transcription factor 4 (OCT4), exhibit a CD44+CD24-/low phenotype (13) and self-renewal ability. They also constitute a minor proportion of neoplastic cells in the tumor microenvironment, which are usually regarded as the tumor-initiating cells (TICs) $(14,15)$. The proportion of TICs in tumors is an important source of metastatic lesions in breast cancer. Current evidence has revealed that cells, which undergo EMT, exhibit stem cell-resembling characteristics $(16,17)$. For example, Mani et al (16) demonstrated that stem-like cells isolated from either mouse or human mammary glands or mammary carcinomas express EMT markers, illustrating an association 
between EMT and the gain of epithelial stem cell properties. Wang et al (18) revealed that fusobacterium nucleatum produced CSC characteristics by activating IL-6/STAT3 and eliciting EMT-resembling activation. Furthermore, EMT induced cancer cell mesenchymal characteristics and promoted cancer cells to gain stemness $(19,20)$. In addition, EMT induces invasion and dissemination of tumor cells, and also assists CSCs to invade to distant organs, leading to cancer distant metastasis $(21,22)$. Numerous studies have demonstrated that poor outcomes of ovarian cancer therapies, i.e., metastasis and relapse, are usually due to a small proportion of CSCs that have escaped from the primary cancer lesion $(23,24)$. Thus, CSC-target therapy is a promising strategy to conquer ovarian cancer metastasis and relapse.

Traditional Chinese Medicine has important roles and has shown good therapeutic efficiency in the prevention of various diseases. In recent years, numerous bioactive small molecular drugs extracted from herbal medicine have been widely investigated in diseases, such as cancer (25-28). Emodin, which can be isolated from several Chinese herbs, including Rheum palmatum $L$ and Poly gonum cuspidatum, has been reported to possess numerous bioactivities in regulating resistance to oxidation and blood glucose levels, in addition to having anti-bacterial properties (29-31). The use of emodin in Traditional Chinese Medicine is 'attack stagnation, clear heat and dampness, purge fire, cool blood, remove blood stasis and detoxification' (32). In addition, according to recent studies, emodin significantly inhibited colon cancer, prostate cancer, breast cancer and skin cancer cells by triggering cancer cell apoptosis $(29,33,34)$. However, the anti-proliferation and anti-metastasis effect of emodin on ovarian cancer has been rarely studied. Therefore, the antitumor effect of emodin in vitro and in vivo was investigated in the present study. In addition, the corresponding mechanism was also analyzed to provide evidence for the therapeutic application of emodin in ovarian cancer.

\section{Materials and methods}

Cell lines and cell culture. The human ovarian cancer cell lines, SK-OV-3 and A2780 were cultured in RPMI-1640 (Gibco; Thermo Fisher Scientific, Inc.), and PA-1 were cultured in DMEM (Gibco; Thermo Fisher Scientific, Inc.), containing 10\% (FBS) (Gibco; Thermo Fisher Scientific, Inc.), were purchased from American Type Culture Collection. All the cells were cultured at $37^{\circ} \mathrm{C}$ in a humidified atmosphere containing $5 \% \mathrm{CO}_{2}$. Emodin was purchased from Sigma-Aldrich (Merck KGaA), dissolved in dimethyl sulfoxide (DMSO) and stored at $-20^{\circ} \mathrm{C}$ for further use. The antibodies against $\mathrm{N}$-cadherin (cat. no. 13116; 1:1,000), E-cadherin (cat. no. 14472, dilution, 1:1,000), vimentin (cat. no. 5741; 1:1,000), CD133 (cat. no. 64326; 1:1,000), OCT4 (cat. no. 2840; $1: 1,000)$ and $\beta$-actin (cat. no. 3700; 1:1,000) were purchased from Cell Signaling Technology, Inc.

Cell proliferation assay. Briefly, suspensions of the SK-OV-3, A2780 and PA-1 cell lines (2,000-5,000 cells/100 $\mu \mathrm{l})$ were added to 96-well microplates. Approximately $12 \mathrm{~h}$ later, the cells were treated with different concentrations $(0,2.5,5,10,20,40$ and $80 \mu \mathrm{M})$ of emodin for 24,48 or $72 \mathrm{~h}$. Then, the cell culture medium in each well was replaced with $20 \mu 1$ MTT solution $(5 \mathrm{mg} / \mathrm{ml})$ and the samples were incubated at $37^{\circ} \mathrm{C}$ for a further 2-4 h. Subsequently, $150 \mu \mathrm{l}$ DMSO was added to each well to dissolve the formazan crystal produced by the living cells. Finally, the optical density of each well was measured using a Spectra MAX M5 microplate spectrophotometer (Molecular Devices, LLC) at $570 \mathrm{~nm}$. At least three independent experiments were performed.

Colony formation assay. The clonogenic ability of the ovarian cancer cell lines was assessed using the colony formation assay following treatment with emodin. Briefly, the ovarian cancer cell lines (400-600 cells/well) were seeded in 6-well plates and cultured for a further $12 \mathrm{~h}$. The cells were treated to various doses of emodin $(0,5,10,20$ and $40 \mu \mathrm{M})$ and cultured for a further 12 days. The cell culture medium was replaced with fresh medium containing corresponding concentrations of emodin every 3 days. Subsequently, the culture medium was discarded, and the cell colonies were washed three times with PBS, fixed with methanol for $15 \mathrm{~min}$ at $25^{\circ} \mathrm{C}$ and stained with $0.5 \%$ crystal violet for $20 \mathrm{~min}$ at $25^{\circ} \mathrm{C}$. Finally, the number of cell colonies ( $>30$ cells) was counted manually and images were captured using an inverted microscope (Axiovert 200; Zeiss AG).

Wound healing assay. The ovarian cancer cell lines were seeded in 6-well plates and cultured for $12 \mathrm{~h}$. When the monolayer of the cells reached $80 \%$ confluence, the wound was created using a sterile $200 \mu 1$ micropipette tip. Then, the cells were washed with fresh medium and cultured in medium (with $0.5 \%$ FBS) and different concentrations of emodin $(0,10,20$ and $40 \mu \mathrm{M})$ for $48 \mathrm{~h}$. Next, the cell culture medium was discarded, the cells were washed three times with PBS, then images were captured using a light microscope (Axiovert 200; Zeiss AG). The migration rates of the treated cells were quantified using the following equation: Migration rate $=\left(1-\mathrm{W}_{0} / \mathrm{W}_{\text {emodin }}\right) /\left(1-\mathrm{W}_{0} / \mathrm{W}_{\text {ctrl }}\right)$, where $\mathrm{W}_{0}$ represents the width of the wound at $0 \mathrm{~h} ; \mathrm{W}_{\text {ctrl }}$ represents the width of the wound at $48 \mathrm{~h}$ in the control group and $\mathrm{W}_{\text {emodin }}$ represents the width of the wound at $48 \mathrm{~h}$ in emodin treatment group. The migration rate of the control cells was regarded as $100 \%$.

Transwell and Matrigel assays. For the analysis of the migratory effect of emodin, $1 \times 10^{5}$ SK-OV-3, A2780 and PA-1 cells, suspended in $100 \mu \mathrm{l}$ serum-free medium, were added to the upper chamber, while $600 \mu \mathrm{l}$ complete medium, containing $10 \%$ FBS, was added to the bottom chamber. Different concentrations of emodin $(0,10,20$ and $40 \mu \mathrm{M})$ were added to medium in the upper chambers. After migration for $\sim 48 \mathrm{~h}$ at $25^{\circ} \mathrm{C}$, non-migratory cells remaining in the upper chamber were discarded using a cotton swab and the migrated cells located on the bottom of membrane were washed, fixed with methanol for $15 \mathrm{~min}$ at $25^{\circ} \mathrm{C}$, then stained with $0.5 \%$ crystal violet for $20 \mathrm{~min}$ at $25^{\circ} \mathrm{C}$. Images of the migrated cells were captured using a light microscope and five random fields of view were counted to compare the migration rate.

A Matrigel assay was performed to determine the invasion ability of the cells treated with emodin. Briefly, the upper surface of a 24-well Transwell plate (MilliporeSigma) was pre-coated with $\sim 70 \mu$ l Matrigel (BD Biosciences) diluted 
(1:4) in serum-free cell culture medium. After Matrigel polymerization for $1 \mathrm{~h}$ at $37^{\circ} \mathrm{C}, 100 \mu \mathrm{l}$ serum-free medium, containing $1 \times 10^{5}$ SK-OV-3, A2780 and PA-1 cells was added to upper chamber, while $600 \mu \mathrm{l}$ the complete medium was added to the lower chamber, which served as a chemoattractant. Different concentrations of emodin $(0,10,20$ and $40 \mu \mathrm{M})$ were added to both the upper and lower chambers. After invasion for $48 \mathrm{~h}$, the non-invasive cells remaining in the upper chamber were discarded using a cotton swab and the invasive cells on the bottom chamber were washed, fixed with methanol for $15 \mathrm{~min}$ at $25^{\circ} \mathrm{C}$, then stained with $0.5 \%$ crystal violet or $20 \mathrm{~min}$ at $25^{\circ} \mathrm{C}$. The images of the invasive cells were captured using a light microscope and five random fields of view were selected to calculate the invasive rate.

Western blot analysis. To investigate the effect of emodin on relevant signaling pathways, the changes in the protein expression level of key proteins in the SK-OV-3, A2780 and PA-1 cell lines were evaluated using western blot analysis. After treatment with the indicated concentrations of emodin $(0,10,20$ and $40 \mu \mathrm{M})$ for $48 \mathrm{~h}$, the SK-OV-3, A2780 and PA-1 cell lines were harvested and lysed with RIPA buffer (Beyotime Institute of Biotechnology) to obtain the total protein. Concentration of total protein was determined by BCA method. An equal amount of protein ( $30 \mathrm{ug})$, from the differently treated samples were separated using SDS-PAGE (10\%), then transferred onto PVDF membranes (Amersham Bioscience; Cytiva). The membranes were blocked with skimmed milk (5\% in TBST buffer (Tween-20, 0.5\%) for $1 \mathrm{~h}$ at $37^{\circ} \mathrm{C}$ and incubated with the primary antibodies overnight at $4^{\circ} \mathrm{C}$, then incubated with the corresponding secondary antibodies (horseradish peroxidase-conjugated goat Anti-Rabbit IgG H\&L; cat. No.: ab205718, dilution: 1:10,000, Abcam) at $37^{\circ} \mathrm{C}$ for $2 \mathrm{~h}$. Immunoreactive protein bands were visualized and detected using an enhanced chemiluminescence kit (MilliporeSigma). The $\beta$-actin antibody was used as a loading control. Protein expression levels were determined by ImageJ (v 1.8.0, National Institutes of Health).

Mouse and tumor model. All the animal experiments were approved by the Institutional Animal Care and Treatment Committee of Gannan Medical University (Jiangxi, China) and were conducted according to the approved guidelines. All mice (Balb/c nude; female; 6-8 weeks old; weight, 18-20 g) used in the experiments were purchased from Beijing HFK Bioscience Co. Ltd. The mice were kept in a specific-pathogen-free condition facility, in an air-conditioned room at $25 \pm 2^{\circ} \mathrm{C}$, with a relative humidity of $40-70 \%$, and a 12-h light/dark cycle, with free access to food and water. A total of 18 female mice (6-8 weeks old; weight, $18-20 \mathrm{~g}$ ) were subcutaneously injected with $100 \mu \mathrm{l}$ suspension (normal saline) of SK-OV-3 cells $\left(0.5 \times 10^{7}\right.$ cells per mouse). Several days later, the injected site (right flank) a successful mouse tumor model was established if there were noticeable signs of growth. When the tumor volume reached $\sim 100 \mathrm{~mm}^{3}$, the mice were randomly divided into three groups (vehicle, and 20 and $40 \mathrm{mg} / \mathrm{kg}$ emodin; $\mathrm{n}=6$ ). The mice were intraperitoneally administrated with 20 and $40 \mathrm{mg} / \mathrm{kg}$ emodin or vehicle once daily. The tumor volume and body weight of the mice was measured and recorded every 3 days during the treatment process, and the tumor volume was calculated as follows: Volume $\left(\mathrm{mm}^{3}\right)=\mathrm{LxW}^{2} / 2$, where $\mathrm{L}$ $(\mathrm{mm})$ represents the length of the tumor and $\mathrm{W}(\mathrm{mm})$ represents the width of the tumor. All the mice were sacrificed with $\mathrm{CO}_{2}(70 \%$ volume displacement rate) at the endpoint of the experiment, which was defined by the tumor volume $\left(\sim 1,500 \mathrm{~mm}^{3}\right)$ in the control group. Tumor inhibitory rate $(\%)=(\mathrm{V}($ emodin $)-\mathrm{V}($ vehicle $)) / \mathrm{V}($ vehicle $)$, where $\mathrm{V}$ represents tumor volume. The tumors from each group were collected, weighted and fixed with $4 \%$ paraformaldehyde for $48 \mathrm{~h}$ at $25^{\circ} \mathrm{C}$ for further evaluation. In addition, the major organs (heart, liver, spleen, the lungs and the kidneys) from the mice in each group were collected, fixed in $4 \%$ paraformaldehyde for $24 \mathrm{~h}$ at $25^{\circ} \mathrm{C}$, then sectioned $(3-5 \mu \mathrm{m})$ for hematoxylin and eosin staining. The sections were stained with hematoxylin $(1 \%)$ and eosin $(1 \%)$ at $25^{\circ} \mathrm{C}$ for $2-5 \mathrm{~min}$. Finally, sections were photographed by light microscope.

Immunohistochemistry (IHC). The collected tumor tissue samples were fixed in $4 \%$ paraformaldehyde for $24 \mathrm{~h}$ at $25^{\circ} \mathrm{C}$, embedded in paraffin and sliced into thin sections $(3-5 \mu \mathrm{m})$. After dewaxing and rehydration, the sections were incubated with $3 \%$ hydrogen peroxide at $25^{\circ} \mathrm{C}$ for 30 min to block the endogenous peroxidase activity, then treated with $5 \%$ bovine serum albumin (Thermo Fisher Scientific, Inc.) at $37^{\circ} \mathrm{C}$ for $30 \mathrm{~min}$ to block non-specific binding. Subsequently, the sections were incubated with primary antibodies against Ki67 (cat. no. ab15580: 1:500), CD133 (cat. no. ab278053; 1:1,000), and cleaved caspase3 (cat. no. ab32042; 1:1,000; all Abcam) overnight at $4^{\circ} \mathrm{C}$. The sections were washed with PBS 3 or 4 times, incubated with the biotinylated secondary antibody (Abcam, ab64256, dilution: $1: 1,000$ ) at $37^{\circ} \mathrm{C}$ for $1 \mathrm{~h}$, then treated with streptavidin horseradish peroxidase at $37^{\circ} \mathrm{C}$ for 20-30 min. Images were captured under a light microscope (Axiovert 200; Zeiss AG). Positive stained cells were calculated by Image J (V1.8.0, National Institutes of Health) from the average of four random fields of view.

Pulmonary metastasis tumor mouse model. A total of 18 mice were intravenously injected with a $100 \mu \mathrm{l}$ suspension SK-OV-3 cells in saline ( $1 \times 10^{6}$ cells per mouse) via the tail vein to produce an experimental lung metastasis tumor mouse model. The mice were randomly divided into three groups (vehicle, and 20 and $40 \mathrm{mg} / \mathrm{kg}$ emodin groups; $\mathrm{n=6}$ ) 2 days following injection and were intraperitoneally injected with 20 or $40 \mathrm{mg} / \mathrm{kg}$ emodin, or vehicle once daily. The mice from the experimental and control groups were euthanized on day 18, and their lung tissues were isolated, weighted and collected for observation of visible metastatic nodules. The dots on the lung surface were counted manually and confirmed as ovarian cancer metastases.

At the study endpoint, the lung tissues from each group were collected and prepared as single-cell suspensions using mechanic dispersion with surgical scissors and enzymatic method of I-collagenase $(1 \mathrm{mg} / \mathrm{ml})$. Then, $1 \times 10^{6}$ freshly prepared cells were suspended in $100 \mu \mathrm{l}$ PBS and stained with different combinations of FITC-CD24 (Biolegend, cat. no. 311103) and APC-CD44 (Biolegend, cat. no. 103011). The cells were analyzed using flow cytometry (FACS Canto II, BD Biosciences) and the data were analyzed using 
A
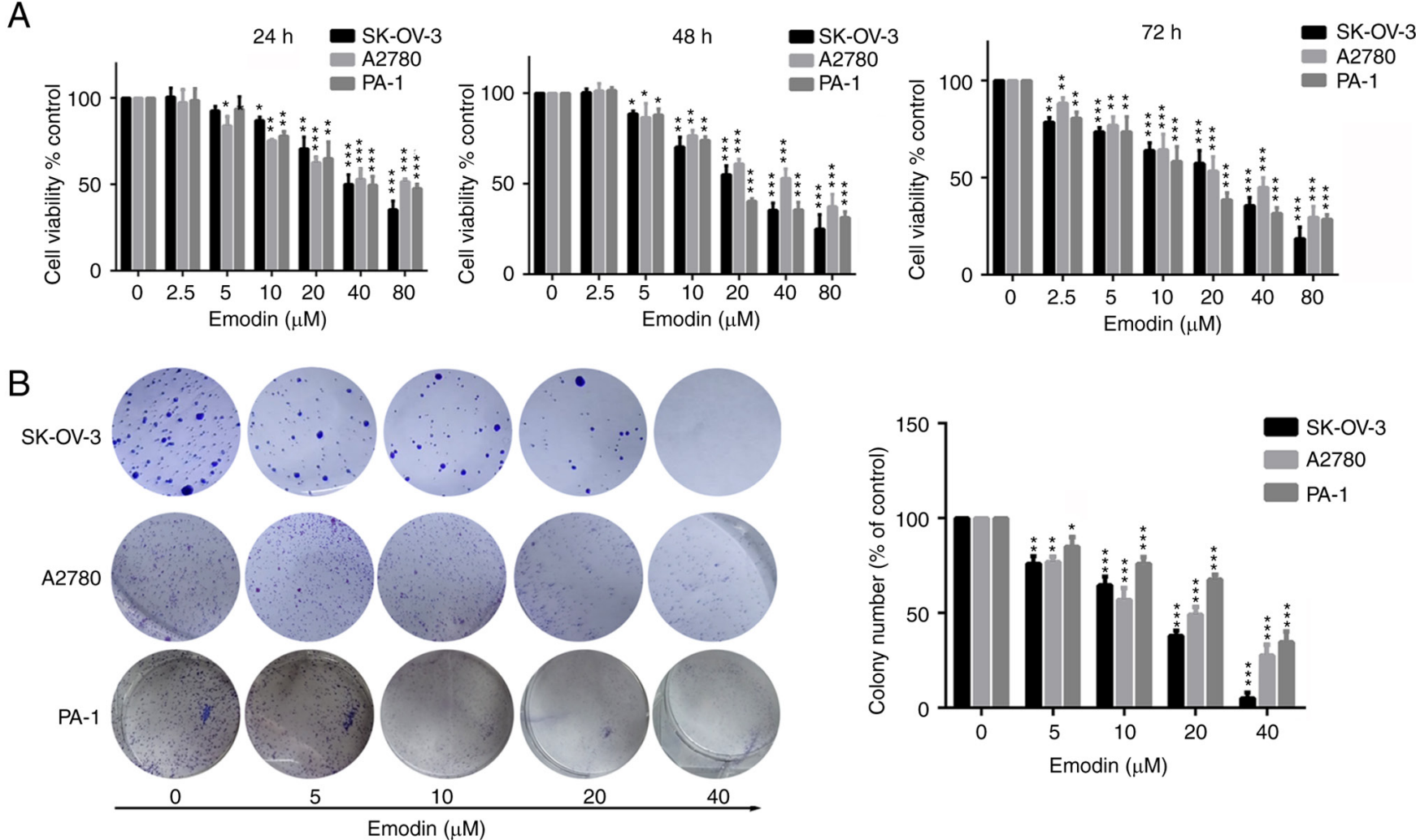

Figure 1. Emodin inhibits the proliferation of ovarian cancer cells. (A) Viabilities of the SK-OV-3, A2780 and PA-1 cell lines were evaluated using a MTT assay following treatment with different concentrations of emodin for 24, 48 and $72 \mathrm{~h}$. (B) Proliferation of the SK-OV-3, A2780 and PA-1 cell lines were assessed using a colony formation assay following treatment with different concentrations of emodin. ${ }^{*} \mathrm{P}<0.05,{ }^{* *} \mathrm{P}<0.01,{ }^{* * *} \mathrm{P}<0.001$ vs. control group $(0 \mu \mathrm{M})$.

FlowJo software (FlowJo LLC, V7.6.1). The proportions of $\mathrm{CD} 44^{+} / \mathrm{CD} 24-$ cells were considered to be ovarian CSCs.

Statistical analysis. The data are presented as the mean \pm standard deviation of at least of three independent experiments. SPSS v16.0 (SPSS, Inc.) software was used to perform the statistical analyses. One-way ANOVA followed by Dunnett's or Tukey's post hoc test was used for multi-group comparisons. $\mathrm{P}<0.05$ was considered to indicate a statistically significant difference.

\section{Results}

Emodin inhibits the viability and proliferation of ovarian cancer cell lines. The SK-OV-3, A2780 and PA-1 cell lines were used to evaluate the antitumor effect of emodin on ovarian cancer. Firstly, the time- and dose-dependent effect of emodin on these cells was performed by treating the cells with different concentrations of emodin $(0,2.5,5,10$, $20,40$ and $80 \mu \mathrm{M})$ for various time points $(24,48$ and $72 \mathrm{~h}$ ). As shown in Fig. 1A, the SK-OV-3, A2780, and PA-1 cell lines treated with 10-20 $\mu \mathrm{M}$ emodin for $24 \mathrm{~h}$ exhibited weakened viability $(\mathrm{P}<0.05)$. However, after treatment for 48 and $72 \mathrm{~h}$, cell viability was significantly $(\mathrm{P}<0.01)$ suppressed. In addition, treatment with 40 and $80 \mu \mathrm{M}$ emodin significantly reduced the viability of the SK-OV-3, A2780 and PA-1 cell lines at 24,48 and $72 \mathrm{~h}$. This suggests that emodin exhibited a time- and dose-dependent ( $>2.5 \mu \mathrm{M}$ emodin) effect in ovarian cancer cell lines. To further investigate whether emodin could suppress the proliferation ability of the ovarian cancer cell lines, a colony formation assay was performed after the cells were treated with emodin. As shown in Fig. 1B, the number of the colonies in the SK-OV-3, A2780 and PA-1 cell lines treated with emodin was significantly reduced in a dose-dependent manner. In addition, emodin significantly inhibited the number of colonies in the SK-OV-3, A2780 and PA-1 cell lines in comparison to the control group. Taken together, these results suggest that emodin significantly inhibited cell viability and colony formation in the ovarian cancer cell lines.

Emodin inhibits ovarian cancer cell migration and invasion by affecting EMT. A wound healing assay was performed using the SK-OV-3, A2780 and PA-1 cell lines to investigate the in vitro anti-migration ability of emodin in ovarian cancer cells. As displayed in Fig. 2A and B, emodin significantly inhibited the wound healing rate of the SK-OV-3 and A2780 cell lines in a dose-dependent manner, suggesting an anti-migratory effect of emodin in ovarian cancer cells. In addition, Transwell and Matrigel assays were performed to further investigate the anti-migratory and anti-invasive abilities of emodin in ovarian cancer cells. The results demonstrated that the migration abilities of the SK-OV-3, A2780 and PA-1 cell lines were significantly suppressed in the presence of emodin compared with that in the control group (Fig. 2C and D). As indicated in Fig. 2E and F, treatment with different concentrations of emodin distinctly inhibited the invasive abilities of the SK-OV-3, A2780 and PA-1 cell lines. Furthermore, as there 
A
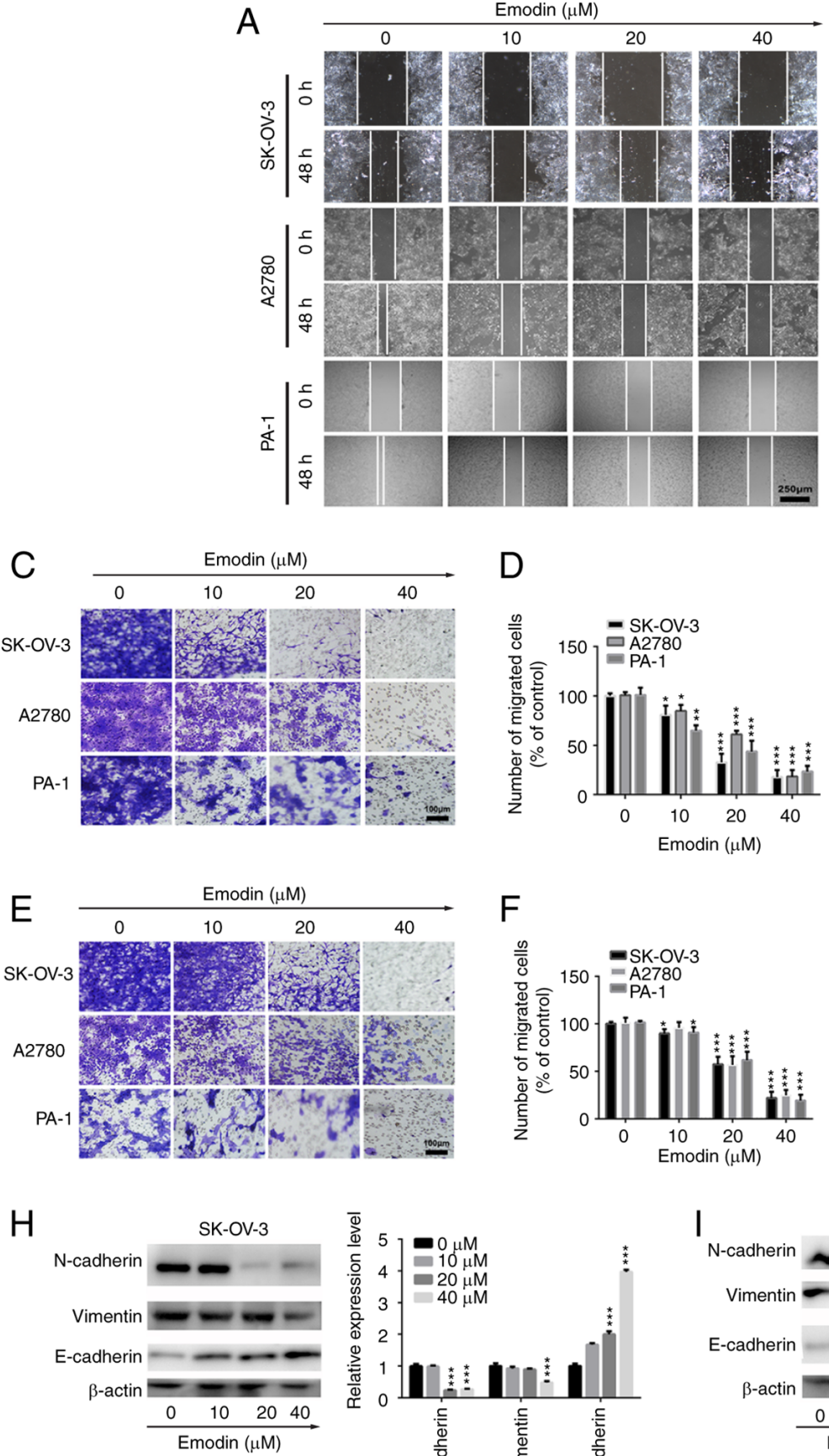

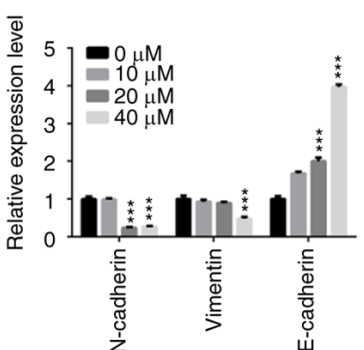

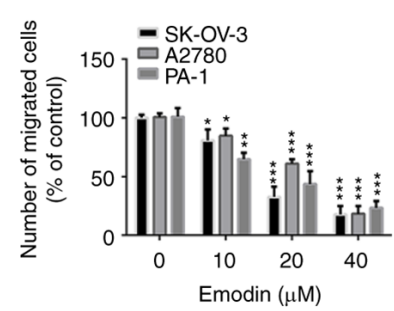

$\mathrm{F}$

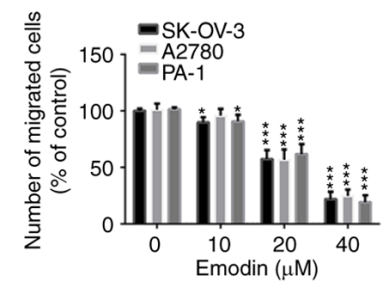

B
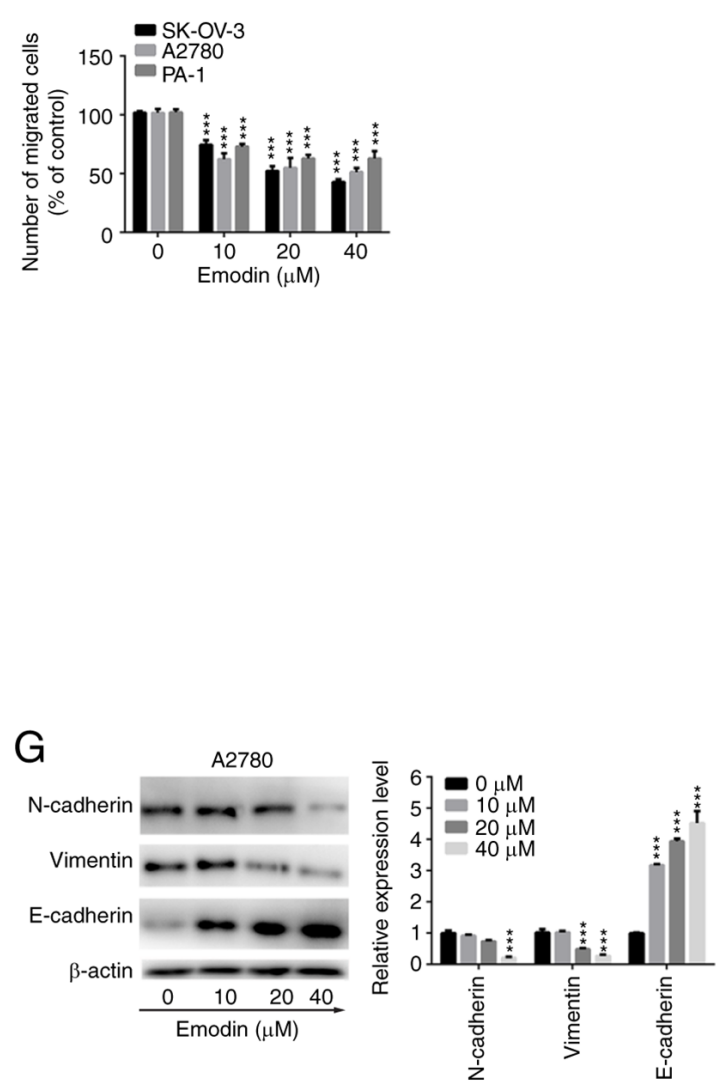

Figure 2. Emodin suppresses the migration and invasion abilities of the ovarian cancer cells via EMT. (A) Migratory ability of the ovarian cancer cells was evaluated using a wound healing assay following treatment with different concentrations of emodin and the results were (B) statistically analyzed. (C) Migration of the ovarian cancer cells was evaluated using a Transwell assay following treatment with emodin and the results were (D) statistically analyzed. (E) Invasion of the ovarian cancer cells was evaluated using a Matrigel assay following treatment with emodin and the results were (F) statistically analyzed. Changes in the protein expression levels of EMT-associated proteins in the (G) SK-OV-3, (H) A2780 and (I) PA-1 cell lines were measured using western blot analysis following treatment with different doses of emodin. ${ }^{*} \mathrm{P}<0.05,{ }^{* *} \mathrm{P}<0.01,{ }^{* * * *} \mathrm{P}<0.001$ vs. control group $(0 \mu \mathrm{M})$.

is an association between cancer cell migration and invasion, and EMT (35), it was investigated whether emodin inhibited the migration and invasion abilities of the ovarian cancer cells via EMT. As demonstrated in Fig. 2G-I, the protein expression levels of $\mathrm{N}$-cadherin and vimentin were decreased in the SK-OV-3, A2780 and PA-1 cell lines following treatment with emodin, whereas the protein expression levels of E-cadherin were increased, indicating EMT was affected in the ovarian cancer cell lines. Taken together, these results suggest that
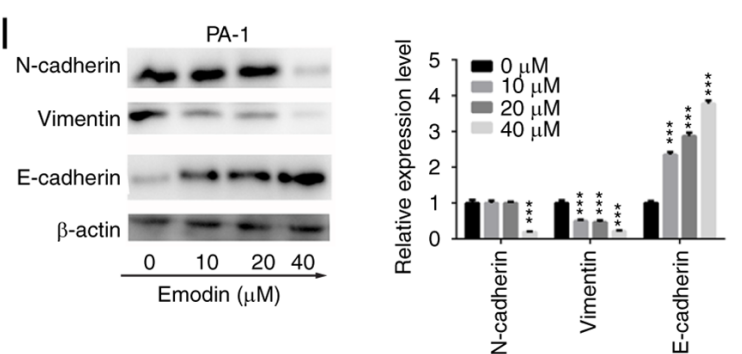

emodin suppressed the migration and invasion abilities of the ovarian cancer cells by affecting EMT.

Emodin significantly inhibits tumor growth in a xenograft model of human ovarian cancer. To investigate whether the antitumor activity of emodin in vivo was consistent with its anti-proliferation effect in vitro, SK-OV-3 tumor-bearing mice were treated with different doses of emodin (20 and $40 \mathrm{mg} / \mathrm{kg}$ ) for 18 days following injection of the cells. 
A
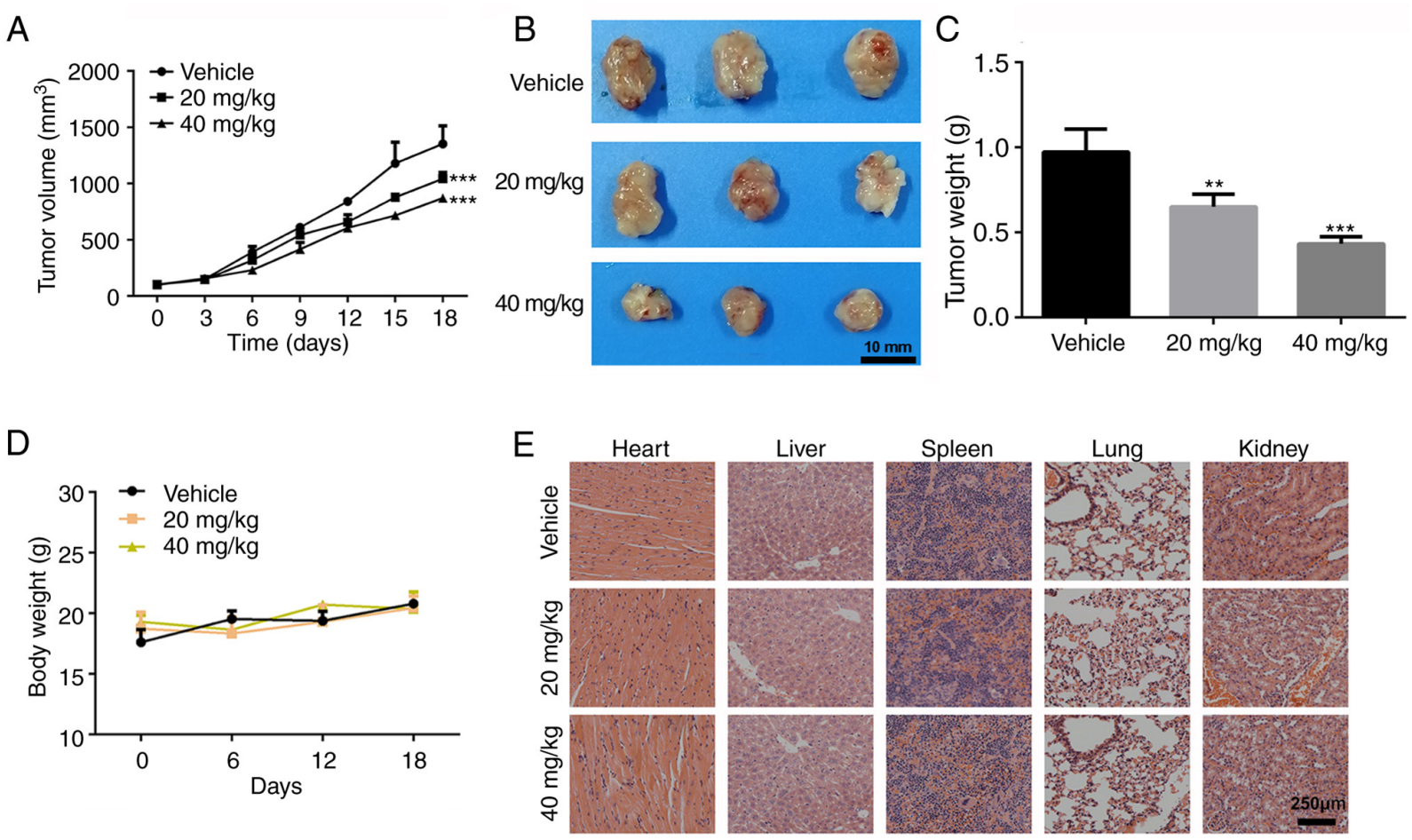

Figure 3. Emodin reduces growth of ovarian cancer in a tumor mouse model. (A) Growth curve of the ovarian tumors during the mouse model. (B) Representative images of isolated tumors at the endpoint of the animal experiment. (C) Weight of the tumors from each group at the endpoint of the in vivo study. (D) Changes in body weight during the mouse tumor model. (E) Pathological evaluation of major organs in each group at the endpoint of in vivo experiment. ${ }^{* *} \mathrm{P}<0.01$, ***** $\mathrm{P}<0.001$ vs. control group (vehicle group).

As shown in Fig. 3A, tumor growth was significantly reduced following treatment with 20 and $40 \mathrm{mg} / \mathrm{kg}$ emodin compared with that in the control group. Mice were sacrificed at the study endpoint, and tumors from each group were isolated. The tumor size and average weight in the 20 and $40 \mathrm{mg} / \mathrm{kg}$ treatment groups were notably smaller compared with that in the control group (Fig. 3B and C). Furthermore, no adverse effects, such as toxic death, skin ulceration and body weight loss were observed, during the treatment of the SK-OV-3 tumor-bearing mice with emodin. The body weight of the mice was not significantly different between the three treatment groups (Fig. 3D). In addition, after pathological evaluation of the major organs (heart, liver, spleen, the lungs and the kidneys), no notable pathological changes were found in the emodin treatment groups, suggesting that emodin is safe to use in vivo (Fig. 3E).

The antitumor effect of emodin was also analyzed using IHC from the tumor sections of each group. As displayed in Fig. 4A and B, increased expression of cleaved-caspase 3 and decreased number of Ki-67 positive cells was observed in the emodin treatment groups. Furthermore, compared with that in the control group, CD133, a key marker of ovarian CSCs (36), was significantly downregulated, suggesting a reduction in the development of ovarian CSCs following treatment with emodin.

Subsequently, western blot analysis was used to verify the anti-CSC effect of emodin. As demonstrated in Fig. 4C and D, the protein expression level of E-cadherin was increased following treatment with emodin, whereas the expression level of N-cadherin was decreased, suggesting that emodin inhibited EMT. In addition, the stemness of the tumors in the emodin treatment groups was significantly suppressed, as evidenced by the decrease in protein expression level of OCT4, which has been validated as a master regulator in the maintenance of the cancer stem-like phenotype (37).

Emodin reduces pulmonary metastasis of ovarian cancer cells by killing cancer stem cells. A pulmonary metastasis mouse model was established using SK-OV-3 cells to evaluate the anti-metastasis properties of emodin. Following treatment with emodin for 18 days, the mice were sacrificed, the weight of the lung tissues was measured, and the number of metastatic lung nodules were counted. As indicated in Fig. 5A and B, compared with that in the control group, the number of metastatic nodules on the lung was markedly reduced in the emodin treatment groups. In addition, the weight of the lungs in the emodin treatment groups was significantly inhibited compared with that in the control group.

It is reported that poor survival and distant metastasis in patients with ovarian cancer are caused by the renewal of CSCs, and increased CSCs have been associated with tumor recurrence or relapse, distant metastasis and chemoresistance $(38,39)$. As aforementioned, the protein expression of the CSC markers, CD133 and OCT4 was significantly decreased in the emodin treatment groups. Various studies have indicated that the $\mathrm{CD} 44^{+} / \mathrm{CD} 24^{-}$population may represent stem cell-like properties of ovarian cancer cells (40-42). To further evaluate whether emodin inhibited lung metastasis of ovarian cancer cells by destruction of the ovarian CSCs, flow cytometry was used to measure the proportion of ovarian CSCs (CD44 $\left.{ }^{+} / \mathrm{CD} 24^{-}\right)$in metastatic lung tissues following emodin treatment for 18 days. As shown in Fig. 5D and E, 
A
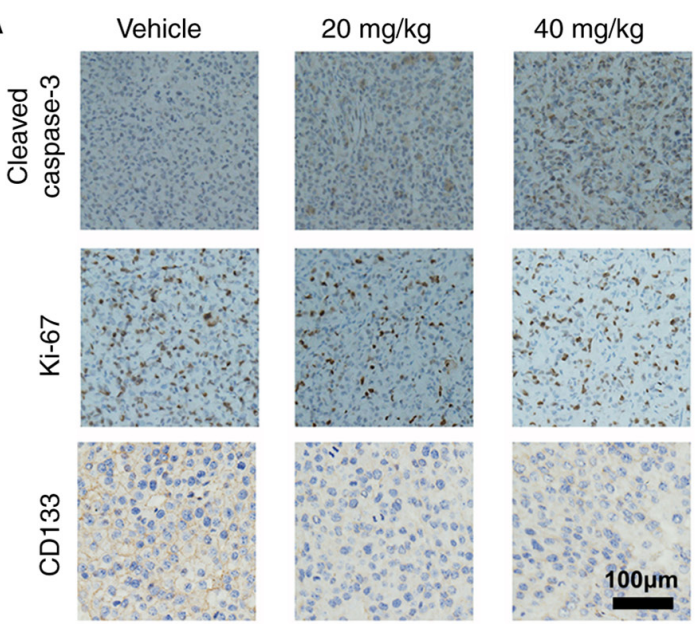

C

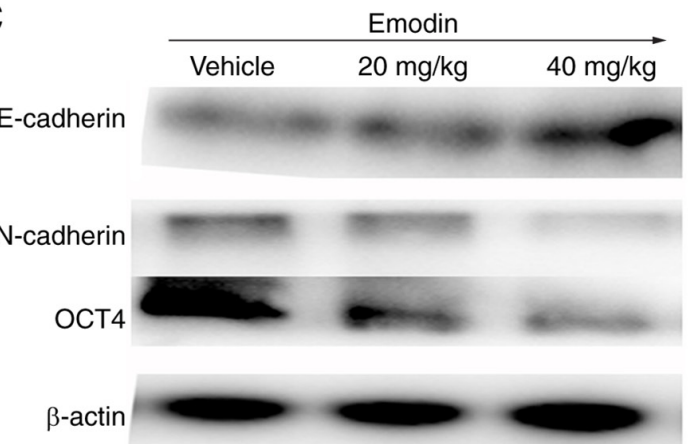

B

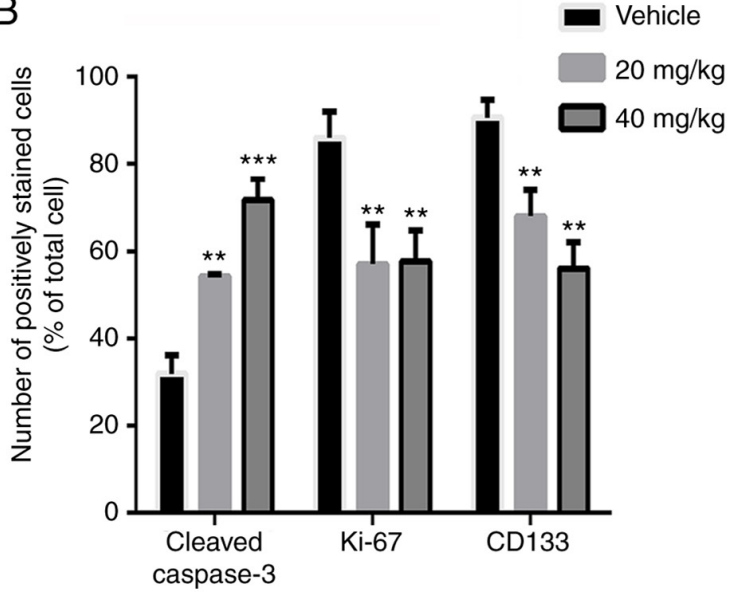

$\mathrm{D}$

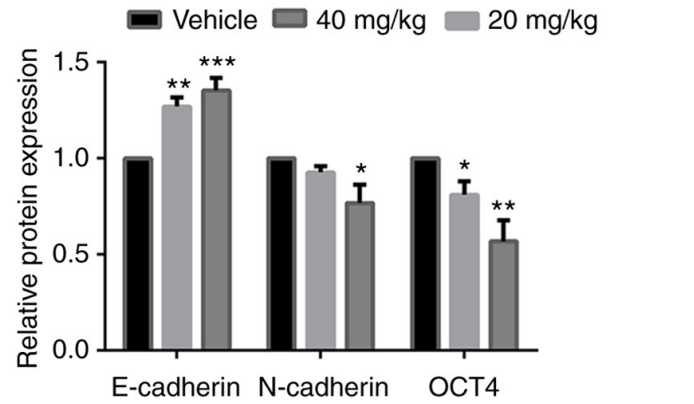

Figure 4. Emodin inhibits ovarian cancer growth by reducing EMT. (A) Immunohistochemical analysis of cleaved-caspase 3, Ki-67 and CD133 in tumor section from each group of the tumor mouse model and the results were (B) statistically analyzed. (C) Changes in the protein expression level of EMT-associated proteins (E-cadherin and N-cadherin) and the cancer stem cell biomarker (OCT4) in tumor tissues was measured using western blot analysis and the results were analyzed using (D) densitometry and statistically analyzed. OCT4, octamer binding transcription factor 4 . ${ }^{*} \mathrm{P}<0.05,{ }^{* * *} \mathrm{P}<0.01,{ }^{* * * *} \mathrm{P}<0.001 \mathrm{vs}$. control group (vehicle group).
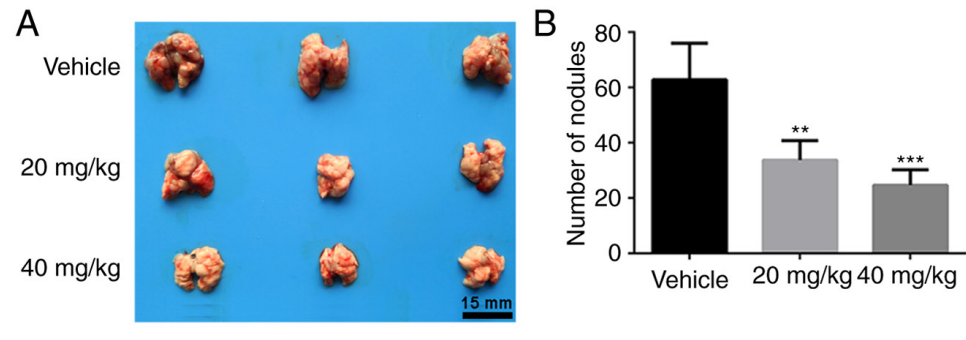

D

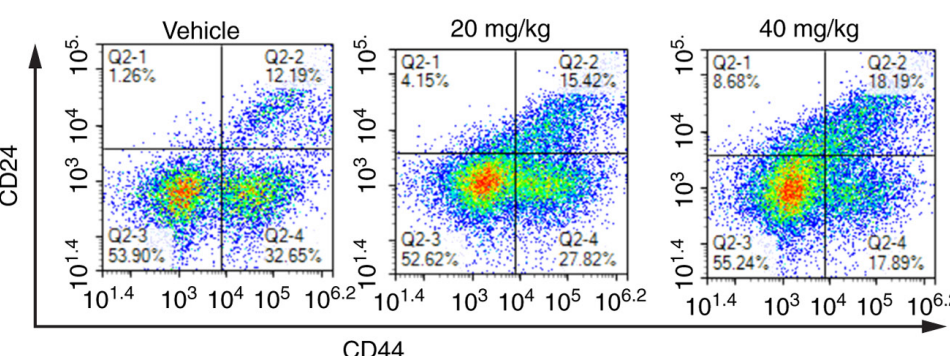

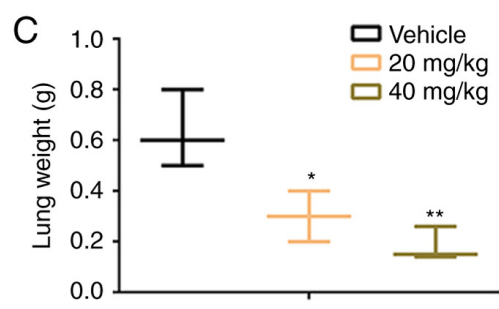

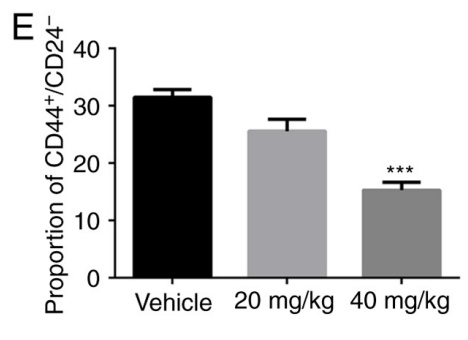

Figure 5. Emodin suppresses pulmonary metastasis of ovarian cancer by affecting the development of ovarian CSCs. (A) Representative images of the lungs from the three treatment groups and (B) numbers of metastatic nodules in the lungs were counted. (C) Weight of the lungs in each group was recorded at the end of the experiment. (D) The proportion of ovarian CSCs $\left(\mathrm{CD} 44^{+} / \mathrm{CD} 24^{-}\right)$in the lung tissues from each group was measured using flow cytometry and the results were (E) statistically analyzed. ${ }^{*} \mathrm{P}<0.05,{ }^{* *} \mathrm{P}<0.01,{ }^{* * *} \mathrm{P}<0.001$ vs. control group (vehicle group).

the percentage of $\mathrm{CD} 44^{+} / \mathrm{CD} 24^{-}$cells was $32.65 \pm 2.95 \%$ in the control group, while treatment with 20 and $40 \mathrm{mg} / \mathrm{kg}$ emodin significantly reduced the proportion of $\mathrm{CD} 44^{+} / \mathrm{CD} 24$ cells to $27.82 \pm 3.21$ and $17.89 \pm 2.76 \%$, respectively $(\mathrm{P}<0.001)$, 
indicating that emodin reduces pulmonary metastasis of ovarian cancer cells by killing cancer stem cells.

\section{Discussion}

Ovarian cancer has the worst prognosis among all types of gynecological malignancies and patients are often only diagnosed at an advanced stage (1). Even though chemotherapy combined with surgery can result in remission in ovarian cancer patients, resistance and distant metastasis remain an obstacle for ovarian cancer therapy (43). Once the patient has relapsed, the outcome of chemotherapy declines significantly, with rapid tumor progression and drug resistance (44). Therefore, the discovery of novel potential drug candidates to prevent tumor metastasis is urgently required.

CSCs have a key role in tumor initiation, invasion, metastasis and therapeutic resistance, as well as in local recurrence following curative resection (45). Therefore, the elimination of CSCs in patients with ovarian cancer is considered to represent an effective strategy for the treatment of this highly refractory malignancy. Furthermore, EMT is an important component of cancer proliferation and distant metastasis (46). The crosstalk between CSC and EMT has been shown to increase cancer cell mesenchymal characteristics on the CSCs and promote cancer cells to gain stemness $(19,20)$. However, there are currently limited agents that preferentially inhibit ovarian CSCs by regulating EMT.

Emodin, which is derived from natural plants, has been reported to exhibit therapeutic effects in several diseases such as hyperlipidemia, anti-viral and anti-liver fibrosis (47-49). Various in vitro studies have demonstrated its effectiveness on the promotion of apoptosis or the inhibition of proliferation in lung, breast and cervical cancer cells (50-52). In the present study, it was found that emodin could reduce the viability of ovarian cancer cells at low concentrations. The anti-proliferation activity of emodin against ovarian cancer cells was verified with MTT and colony formation assays.

Emodin was also found to exhibit an inhibitory effect on migration and invasion in ovarian cancer cells by inhibiting EMT, as evidenced by the decrease in the protein expression level of $\mathrm{N}$-cadherin and vimentin, and the increase in the protein expression level of E-cadherin following treatment with emodin. It has previously been reported that emodin inhibited pancreatic cancer EMT and invasion by increase the expression level of microRNA-1271 (53). It has been demonstrated that emodin inhibited colon cancer cell invasion and migration by suppressing EMT via the $\mathrm{WNT} / \beta$-catenin pathway (54). Emodin could also suppress the proliferation and invasion of colorectal cancer cells by inhibiting VEGFR2 protein expression (55). Therefore, the antitumor effects of emodin were analyzed using a subcutaneous xenograft SK-OV-3 tumor mouse model. The results of the animal experiments suggested that the tumor growth rate and tumor weight were significantly inhibited by the administration of emodin $(40 \mathrm{mg} / \mathrm{kg})$, with an inhibitory rate of $\sim 45 \%$. The effects of emodin $(20 \mathrm{mg} / \mathrm{kg})$ on tumor growth rate and tumor weight were lower than that of $40 \mathrm{mg} / \mathrm{kg}$. Mechanistic analysis demonstrated that emodin reduced the proliferative ability of the tumors and decreased EMT. In addition, decreased protein expression levels of CD133 and Oct 4 were observed in the tumor tissues following treatment with emodin, which indicates that emodin could inhibit the stemness of ovarian cancer cells. Various studies have demonstrated that inhibition of EMT results in impairment of stemness during the reprogramming of somatic cells $(56,57)$. Liu et al $(51)$ reported that emodin reduced breast cancer lung metastasis by suppressing macrophage-induced breast cancer cell EMT and cancer stem cell formation. It has also been reported that fusobacterium nucleatum produced cancer stem cell characteristics by activating IL-6/STAT3 and eliciting EMT-resembling activation (18). However, there is not enough evidence to demonstrate that downregulation of CD133 and OCT4 could directly lead to impairment of stemness of tumor cells. CD133 and OCT4 are only markers of tumor stem cells and emodin was only found to markedly inhibit EMT activity and impair the stemness of tumor cells from the decrease in the expression level of CD133 and Oct4. In future experiments, CD133 and Oct 4 expression will be knocked down or overexpressed using transfection with small inhibiting RNA/overexpression plasmid to further investigate the function of CD133 and Oct4 in the formation of cancer stem cells.

Ovarian cancer, which starts as a local tumor lesion can metastasize to distant organs, including the lymph nodes, the lungs and the breasts (58). The metastatic process of cancer cells from the primary tumor to the distant site is complex, and requires migration from local lesions to blood vessels (59). As emodin reduced the migratory and invasion ability of the ovarian cancer cell lines, the anti-metastasis effect of emodin was investigated using a pulmonary metastasis tumor mouse model. The results showed that administration of emodin, at 20 and $40 \mathrm{mg} / \mathrm{kg}$, significantly inhibited lung metastasis of ovarian cancer, which was consistent with the in vitro experiments. Furthermore, poor outcomes of ovarian cancer therapies are usually caused by a small proportion of CSCs, that have escaped from the primary cancer lesion (60). Thus, elimination of CSCs in the tumor microenvironment could be a promising strategy to conquer ovarian cancer metastasis. In the present study, it was demonstrated that emodin could reduce the proportion of ovarian CSC in metastatic lung tissues, suggesting that emodin suppressed pulmonary metastasis of ovarian cancer.

In summary, the present study provided important information regarding the antitumor activities of emodin in ovarian cancer. Emodin exhibited antitumor and anti-metastasis effects on ovarian cancer by inhibiting EMT and ovarian CSC formation. The results suggest that emodin could serve as a potential drug for treating ovarian cancer and metastasis.

\section{Acknowledgements}

Not applicable.

\section{Funding}

No funding was received.

\section{Availability of data and materials}

The datasets used and/or analyzed during the current study are available from the corresponding author upon reasonable request. 


\section{Authors' contributions}

HML and HYC conceived and designed the study. HML acquired and analyzed the data. HML, HMC and JY interpreted the data and wrote the manuscript. HML and HYC confirm the authenticity of all the raw data. All authors read and approved the final version of the manuscript.

\section{Ethics approval and consent to participate}

All the animal experiments in this study were performed according to the National Institutes of Health guidelines and were approved by the Institutional Animal Care and Use Committee of Gannan Medical University (Jiangxi, China).

\section{Patient consent for publication}

Not applicable.

\section{Competing interests}

The authors declare that they have no competing interests.

\section{References}

1. Kleppe M, Wang T, Van Gorp T, Slangen BF, Kruse AJ and Kruitwagen RF: Lymph node metastasis in stages I and II ovarian cancer: A review. Gynecol Oncol 123: 610-614, 2011.

2. Pakneshan S, Safarpour D, Tavassoli F and Jabbari B: Brain metastasis from ovarian cancer: A systematic review. J Neurooncol 119: 1-6, 2014

3. Ottevanger PB: Ovarian cancer stem cells more questions than answers. Semin Cancer Biol 44: 67-71, 2017.

4. Crosbie EJ, Flaum N, Harkness EF, Clayton RD, Holland C, Martin-Hirsch P, Wood N, Keating P, Woodward ER, Lalloo F, et al: Specialist oncological surgery for removal of the ovaries and fallopian tubes in BRCA1 and BRCA2 pathogenic variant carriers may reduce primary peritoneal cancer risk to very low levels. Int J Cancer 148: 1155-1163, 2020.

5. Zheng $\mathrm{H}$ and Gao YN: Primary debulking surgery or neoadjuvant chemotherapy followed by interval debulking surgery for patients with advanced ovarian cancer. Chin J Cancer Res 24: 304-309, 2012

6. Ducoulombier S, Golfier F, Colomban O, Benayoun D, Bolze PA, Tod M and You B: Modeling CA-125 during neoadjuvant chemotherapy for predicting optimal cytoreduction and relapse risk in ovarian cancer. Anticancer Res 37: 6879-6886, 2017.

7. Wang Y, Herrstedt J, Havsteen H, DePoint Christensen R, Mirza MR, Lund B, Maenpaa J and Kristensen G: A multicenter, non-randomized, phase II study of docetaxel and carboplatin administered every 3 weeks as second line chemotherapy in patients with first relapse of platinum sensitive epithelial ovarian, peritoneal or fallopian tube cancer. BMC Cancer 14 937, 2014.

8. Zhang B, Chen F, Xu Q, Han L, Xu J, Gao L, Sun X, Li Y, Li Y, Qian $M$ and Sun Y: Revisiting ovarian cancer microenvironment: A friend or a foe? Protein Cell 9: 674-692, 2018.

9. Luo Q, Wu T, Wu W, Chen G, Luo X, Jiang L, Tao H, Rong M, Kang S and Deng M: The functional role of voltage-gated sodium channel Nav1.5 in metastatic breast cancer. Front Pharmacol 11: $1111,2020$.

10. Wang W, Wang J, Yan H, Zhang K and Liu Y: Upregulation of USP11 promotes epithelialtomesenchymal transition by deubiquitinating Snail in ovarian cancer. Oncol Rep 41: 1739-1748, 2019.

11. Dai G, Sun B, Gong T, Pan Z, Meng Q and Ju W: Ginsenoside $\mathrm{Rb} 2$ inhibits epithelial-mesenchymal transition of colorectal cancer cells by suppressing TGF-beta/Smad signaling. Phytomedicine 56: 126-135, 2019.

12. Oh E, Hong $\mathbf{J}$ and Yun CO: Regulatory $\mathrm{T}$ cells induce metastasis by increasing Tgf- $\beta$ and enhancing the epithelial-mesenchymal transition. Cells 8: 1387, 2019.
13. van Schaijik B, Davis PF, Wickremesekera AC, Tan ST and Itinteang T: Subcellular localisation of the stem cell markers OCT4, SOX2, NANOG, KLF4 and c-MYC in cancer: A review. J Clin Pathol 71: 88-91, 2018.

14. Fu W, Lei C, Yu Y, Liu S, Li T, Lin F, Fan X, Shen Y, Ding M, Tang Y, et al: EGFR/Notch antagonists enhance the response to inhibitors of the PI3K-Akt pathway by decreasing tumor-initiating cell frequency. Clin Cancer Res 25: 2835-2847, 2019.

15. Hu S, Fu W, Li T, Yuan Q, Wang F, Lv G, Lv Y, Fan X, Shen Y, Lin F, et al: Antagonism of EGFR and Notch limits resistance to EGFR inhibitors and radiation by decreasing tumor-initiating cell frequency. Sci Transl Med 9: eaag0339, 2017.

16. Mani SA, Guo W, Liao MJ, Eaton EN, Ayyanan A, Zhou AY, Brooks M, Reinhard F, Zhang CC, Shipitsin M, et al: The epithelial-mesenchymal transition generates cells with properties of stem cells. Cell 133: 704-715, 2008.

17. Morel AP, Lievre M, Thomas C, Hinkal G, Ansieau S and Puisieux A: Generation of breast cancer stem cells through epithelial-mesenchymal transition. PLoS One 3: e2888, 2008.

18. Wang Q, Yu C, Yue C and Liu X: Fusobacterium nucleatum produces cancer stem cell characteristics via EMT-resembling variations. Int J Clin Exp Pathol 13: 1819-1828, 2020.

19. Čipak Gašparović A, Milković L, Dandachi N, Stanzer S, Pezdirc I, Vrančić J, Sitić S, Suppan C and Balic M: Chronic oxidative stress promotes molecular changes associated with epithelial mesenchymal transition, NRF2, and breast cancer stem cell phenotype. Antioxidants (Basel) 8: 633, 2019.

20. Navas T, Kinders RJ, Lawrence SM, Ferry-Galow KV, Borgel S, Hollingshead MG, Srivastava AK, Alcoser SY, Makhlouf HR, Chuaqui R, et al: Clinical evolution of epithelial-mesenchymal transition in human carcinomas. Cancer Res 80: 304-318, 2020.

21. Hoca M, Becer E, Kabadayi H, Yucecan S and Vatansever HS: The effect of resveratrol and quercetin on epithelial-mesenchymal transition in pancreatic cancer stem cell. Nutr Cancer 72: $1231-1242,2020$

22. Zhou JM, Hu SQ, Jiang H, Chen YL, Feng JH, Chen ZQ and Wen KM: OCT4B1 promoted EMT and regulated the self-renewal of CSCs in CRC: Effects associated with the balance of miR-8064/PLK1. Mol Ther Oncolytics 15: 7-20, 2019.

23. Kenda Suster N and Virant-Klun I: Presence and role of stem cells in ovarian cancer. World J Stem Cells 11: 383-397, 2019.

24. Zhao Y, He M, Cui L, Gao M, Zhang M, Yue F, Shi T, Yang X, Pan Y,Zheng X, et al: Chemotherapy exacerbates ovarian cancer cell migration and cancer stem cell-like characteristics through GLI1. Br J Cancer 122: 1638-1648, 2020.

25. Song L, Chen X, Mi L, Liu C, Zhu S, Yang T, Luo X, Zhang Q, $\mathrm{Lu} \mathrm{H}$ and Liang X: Icariin-induced inhibition of SIRT6/NF- $\mathrm{BB}$ triggers redox mediated apoptosis and enhances anti-tumor immunity in triple-negative breast cancer. Cancer Sci 111: 4242-4256, 2020.

26. Wang C, Wang J, Chen K, Pang H, Li X, Zhu J, Ma Y, Qiu T, Li W, Xie J and Zhang J: Caprylic acid (C8:0) promotes bone metastasis of prostate cancer by dysregulated adipo-osteogenic balance in bone marrow. Cancer Sci 111: 3600-3612, 2020.

27. Zeng A, Liang X, Zhu S, Liu C, Luo X, Zhang Q and Song L: Baicalin, a potent inhibitor of $N F-\kappa B$ signaling pathway, enhances chemosensitivity of breast cancer cells to docetaxel and inhibits tumor growth and metastasis both in vitro and in vivo. Front Pharmacol 11: 879, 2020.

28. Zhang X, Ruan Q, Zhai Y, Lu D, Li C, Fu Y, Zheng Z, Song Y and Guo J: Baicalein inhibits non-small-cell lung cancer invasion and metastasis by reducing ezrin tension in inflammation microenvironment. Cancer Sci 111: 3802-3812, 2020.

29. Cui Y, Chen LJ, Huang T, Ying JQ and Li J: The pharmacology, toxicology and therapeutic potential of anthraquinone derivative emodin. Chin J Nat Med 18: 425-435, 2020.

30. Li Q, Gao J, Pang X, Chen A and Wang Y: Molecular mechanisms of action of emodin: As an anti-cardiovascular disease drug. Front Pharmacol 11: 559607, 2020.

31. Luo N, Fang J, Wei L, Sahebkar A, Little PJ, Xu S, Luo C and Li G: Emodin in atherosclerosis prevention: Pharmacological actions and therapeutic potential. Eur J Pharmacol 890: 173617, 2020.

32. Zhang Y, Zhang M, Hu G, Zhang Z and Song R: Elevated system exposures of baicalin after combinatory oral administration of rhein and baicalin: Mainly related to breast cancer resistance protein (ABCG2), not UDP-glucuronosyltransferases. J Ethnopharmacol 250: 112528, 2020. 
33. Shrimali D, Shanmugam MK, Kumar AP, Zhang J, Tan BK, Ahn KS and Sethi G: Targeted abrogation of diverse signal transduction cascades by emodin for the treatment of inflammatory disorders and cancer. Cancer Lett 341: 139-149, 2013.

34. Song K, Lv T, Chen Y, Diao Y, Yao Q and Wang Y: Emodin inhibits TGF- $\beta 2$ by activating the FOXD3/miR199a axis in ovarian cancer cells in vitro. Oncol Rep 39: 2063-2070, 2018.

35. Dhamija S and Diederichs S: From junk to master regulators of invasion: lncRNA functions in migration, EMT and metastasis. Int J Cancer 139: 269-280, 2016.

36. Caspa Gokulan R and Devaraj H: Stem cell markers CXCR-4 and CD133 predict aggressive phenotype and their double positivity indicates poor prognosis of oral squamous cell carcinoma. Cancers (Basel) 13: 5895, 2021.

37. Liu HL, Tang HT, Yang HL, Deng TT, Xu YP, Xu SQ, Peng L, Wang Z, Fang Q, Kuang XY and Li QS: Oct4 regulates the transition of cancer stem-like cells to tumor endothelial-like cells in human liver cancer. Front Cell Dev Biol 8: 563316, 2020.

38. Lopez de Andres J, Grinan-Lison C, Jimenez G and Marchal JA Cancer stem cell secretome in the tumor microenvironment: A key point for an effective personalized cancer treatment. J Hematol Oncol 13: 136, 2020.

39. Xu J, Su Q, Gao M, Liang Q, Li J and Chen X: Differential expression and effects of Peroxiredoxin-6 on drug resistance and cancer stem cell-like properties in non-small cell lung cancer. Onco Targets Ther 12: 10477-10486, 2019.

40. He QZ, Luo XZ, Wang K, Zhou Q, Ao H, Yang Y, Li SX, Li Y, Zhu HT and Duan T: Isolation and characterization of cancer stem cells from high-grade serous ovarian carcinomas. Cell Physiol Biochem 33: 173-184, 2014.

41. Huang R, Zhu L and Zhang Y: XIST lost induces ovarian cancer stem cells to acquire taxol resistance via a KMT2C-dependent way. Cancer Cell Int 20: 436, 2020.

42. Wang W, Gao Y, Hai J, Yang J and Duan S: HER2 decreases drug sensitivity of ovarian cancer cells via inducing stem cell-like property in an NFKB-dependent way. Biosci Rep 39: BSR20180829, 2019.

43. Corrado G, Salutari V, Palluzzi E, Distefano MG, Scambia G and Ferrandina G: Optimizing treatment in recurrent epithelia ovarian cancer. Expert Rev Anticancer Ther 17: 1147-1158, 2017.

44. Karadimitris A, Chaidos A, Caputo V, Goudevenou K, Ponnusamy K and Xiao X: Myeloma propagating cells, drug resistance and relapse. Stem Cells 33: 3205-3211, 2015.

45. Clarke MF: Clinical and therapeutic implications of cancer stem cells. N Engl J Med 380: 2237-2245, 2019.

46. Saxena K, Jolly MK and Balamurugan K: Hypoxia, partial EMT and collective migration: Emerging culprits in metastasis. Transl Oncol 13: $100845,2020$.

47. He L, Wang C, Zhang Y, Guo C, Wan Y and Li Y: Effect of emodin on hyperlipidemia and hepatic lipid metabolism in zebrafish larvae fed a high-cholesterol diet. Chem Biodivers: e202100675, 2021 doi: 10.1002/cbdv.202100675 (Epub ahead of print).

48. Horvat M, Avbelj M, Duran-Alonso MB, Banjanac M, Petkovic $\mathrm{H}$ and Iskra $\mathrm{J}$ : Antiviral activities of halogenated emodin derivatives against human coronavirus NL63. Molecules 26: 6825, 2021.
49. Zhou Y, Wu R, Cai FF, Zhou WJ, Lu YY, Zhang H, Chen QL, Sun MY and Su SB: Development of a novel anti-liver fibrosis formula with luteolin, licochalcone A, aloe-emodin and acacetin by network pharmacology and transcriptomics analysis. Pharm Biol 59: 1594-1606, 2021 .

50. Gao R, Wu X, Huang Z, Wang B, Li F, Xu H and Ran L: Anti-tumor effect of aloe-emodin on cervical cancer cells was associated with human papillomavirus E6/E7 and glucose metabolism. Onco Targets Ther 12: 3713-3721, 2019.

51. Liu Q, Hodge J, Wang J, Wang Y, Wang L, Singh U, Li Y, Yao Y, Wang D, Ai W, et al: Emodin reduces breast cancer lung metastasis by suppressing Macrophage-induced breast cancer cell Epithelial-mesenchymal transition and cancer stem cell formation. Theranostics 10: 8365-8381, 2020.

52. Tong H, Huang Z, Chen H, Zhou B, Liao Y and Wang Z: Emodin reverses gemcitabine resistance of pancreatic cancer cell lines through inhibition of IKK $\beta / \mathrm{NF}-\kappa \mathrm{B}$ signaling pathway. Onco Targets Ther 13: 9839-9848, 2020.

53. Li N, Wang C, Zhang P and You S: Emodin inhibits pancreatic cancer EMT and invasion by upregulating microRNA1271. Mol Med Rep 18: 3366-3374, 2018.

54. Gu J, Cui CF, Yang L, Wang L and Jiang XH: Emodin inhibits colon cancer cell invasion and migration by suppressing epithelial-mesenchymal transition via the Wnt/beta-catenin pathway. Oncol Res 27: 193-202, 2019.

55. Dai G, Ding K, Cao Q, Xu T, He F, Liu S and Ju W: Emodin suppresses growth and invasion of colorectal cancer cells by inhibiting VEGFR2. Eur J Pharmacol 859: 172525, 2019.

56. Liu SC, Huang CM, Bamodu OA, Lin CS, Liu BL, Tzeng YM, Tsai JT, Lee WH and Chen TM: Ovatodiolide suppresses nasopharyngeal cancer by targeting stem cell-like population, inducing apoptosis, inhibiting EMT and dysregulating JAK/STAT signaling pathway. Phytomedicine 56: 269-278, 2019.

57. Tanabe S, Quader S, Cabral H and Ono R: Interplay of EMT and CSC in cancer and the potential therapeutic strategies. Front Pharmacol 11: 904, 2020.

58. Luo Z, Wang Q, Lau WB, Lau B, Xu L, Zhao L, Yang H, Feng M, Xuan Y, Yang Y, et al: Tumor microenvironment: The culprit for ovarian cancer metastasis? Cancer Lett 377: 174-182, 2016.

59. Bockhorn M, Jain RK and Munn LL: Active versus passive mechanisms in metastasis: Do cancer cells crawl into vessels, or are they pushed? Lancet Oncol 8: 444-448, 2007.

60. Nowicki A, Kulus M, Wieczorkiewicz M, Pieńkowski W, Stefańska K, Skupin-Mrugalska P, Bryl R, Mozdziak P, Kempisty B and Piotrowska-Kempisty H: Ovarian cancer and cancer stem cells-cellular and molecular characteristics, signaling pathways, and usefulness as a diagnostic tool in medicine and oncology. Cancers (Basel) 13: 4178, 2021.

(i) $\odot$ This work is licensed under a Creative Commons Attribution-NonCommercial-NoDerivatives 4.0 International (CC BY-NC-ND 4.0) License. 